\title{
Velocity of Blood flow through uniform rigid artery of human body
}

\author{
Dr.Amarjyoti Goswami*, Sarfraz Ahmed*, Dr.Krishna Gopal Singha\# \\ * Kaziranga Univeraity, Assam, India, \\ \#Karanga Girls' H.S.S., Assam, India.
}

\begin{abstract}
An attempt is made in this analysis to study the flow of blood through a uniform rigid artery with an axial velocity slip condition at vessel wall has been considered by assuming blood a power fluid. It has been observed that the effect of the Hartmann number and the Reynolds number on the velocity field as well as on the wall shear stress is very prominent and when the Hartmann number and Reynolds number increases, the fluid velocity decreases. It also includes Poiseuille fluid models of blood for both slip and no-slip at a wall and onelayered power fluid model and no slip at tube wall as its special cases. The role of slip in influencing the flow variables and physiological implications of this theoretical modeling are discussed in brief.
\end{abstract}

Keywords: Power fluid model, stenosed vessels, Magnetic effect, Reynolds number, Hartmann number Blood flow.

\section{Introduction}

Human blood is a suspension of red cells in a continuous and aqueous substance called plasma (Boyd [5]).Plasma behaves as Newtonian fluid whereas whole blood shows non-Newtonian characteristic (Fung [9]) McDonald [13] has pointed out that in a case of blood vessels with diameter above 250 micrometers, blood may be considered as a Newtonian fluid.Stenosis is formed by substance depositing on vessel walls. Stenosis is formed by substance depositing on vessel walls. A stenosis may lead to partial or total vessel blockage in some instances and therefore poses a serious medical problem. In a human body, physiological fluids present in human systems include digestive juices, sweating, blood, saliva, urine etc. Among the body fluids, the most important is obviously blood which is regarded as a suspension of different cells in a continuous aqueous solution called plasma (Boyd [5]). Plasma behaves as Newtonian fluid whereas, whole blood shows nonNewtonian characteristics (Fung [9]) McDonald [13] has pointed out. But at low shear rates, blood exhibits nonNewtonian behavior .It is well known that non-Newtonian nature of blood significantly influences the flows, particularly in the cases where blood vessels are curved, branching or narrow etc are explained by M.Mustafa.Ahmad Khan[14] M.Mustafa ,T.Hayat,I.Pop and A. Aziz also studied unsteady boundary layer flow of a casson fluid due to an impulsive started moving flat plate[11]. Further, blood has a finite yield stress and Casson's equation can take care of this property which has been reported by many investigators (Cokelet et al. [8], Merril et al. [15], Bhuyan, B.C and Hazarika [2]). Owing to the intermittent pumping of heart muscles it produces a pressure gradient and as a consequence, blood flows through the circulatory systems (Sud, V.K [17] Rathod V.P [18]). Arterial system is also subject to continuous shear stress changes. One of the circulatory diseases is atherosclerosis which happens due to lipid or lime deposits (atheroma) on the inner coat of the arteries. About $75 \%$ of all deaths in the industrialized world are caused due to circulatory disease. The accumulation of lipid, fat and other particles along the inside artery wall lead to narrowing of the vessel. In some cases, it is observed that a so-called stenosis of the vessel with age that means elastic fibers in vessel wall are replaced by more rigid collagen fibers and this process is accompanied by the widening of the arterial lumen (Malik [12]). Also, the diseased areas of blood vessels are characterized by adhesiveness, hardening, loss of elasticity and contraction in the cross-section, found predominantly in the vicinity of beads, bifurcations and other places. As a consequence, there may arise serious complications and disorders in blood supply due to narrowing in the bore of the vessels or widening of the inner wall. Top restore normal blood supply in diseased vessels, it seems, is an introduction of velocity slip at tube wall will be meaningful (Nubar [16], Brunn [7], Biswas and Nath [6]). In order to study the rheology of blood along with its role in the fundamental understanding of many cardiovascular diseases like myocardial infarction, stroke, thrombosis, sickle cell diseases etc. In their models, flow has been considered steady or pulsatile, fluid as a Newtonian one and the boundary condition on no-slip at wall etc. As blood shows some deviations from Newtonian behavior, it seems consideration of blood, in behaving like a non-Newtonian fluid along with slip at vessel wall etc as is done here, will be appropriate and significant from the physiological point of view. The application of magneto hydrodynamics principles in medicine, engineering is of growing interest M.E.Ali[10] . Many investigations have been made on blood flow under the effect of magnetic field. Barnothy [1] has shown that by the application of an external magnetic field the biological systems are greatly affected. Vardanyan [19] showed 
that the application of magnetic field reduces the flow of blood. Bhuyan and Hazarika [2] have investigated the problem of blood flow with effects of slip in arterial stenosis due to presence of transverse magnetic field and they have also observed that the applied magnetic field reduces the velocity of blood through arteries.

\section{Mathematical Formulation Of The Problem}

A two-fluid modeled for blood flow in a uniform tube has been developed in the present problem. The model basically consists of a core of a red cell suspension in the middle layer and the peripheral plasma in the outer layer. The steady laminar flow of an incompressible fluid, assuming it to behave as a power fluid (Fung [9], Bhuyan ,B.C Hazarika [2],[3][4]), through a rigid circular tube is considered. The axial coordinate and velocity are $\hat{z}$ and $\hat{v}$ respectively. $B$ is the applied magnetic field in $\hat{r}$ direction. Flow is governed by the continuity and Navier-Stokes equations and in addition, power constitutive equation the magnetic field applied in $\hat{r}$ a direction the equations in the axial and radial directions in dimensionless form. From the report of Young [20] and Sud [17] considering the axisymmetric laminar steady flow of blood, the general constitutive equation in the case of mild stenosis subject to the addition may, therefore, be written as:

$$
\begin{aligned}
& -\frac{\partial \hat{p}}{\partial \hat{r}}=0 \\
& -\frac{1}{\hat{r}} \frac{\partial \hat{p}}{\partial \hat{\theta}}=0 \\
& -\frac{1}{\rho} \frac{\partial \hat{p}}{\partial \hat{z}}+\frac{\mu}{\hat{r}} \frac{1}{\rho} \frac{\partial}{\partial \hat{r}}\left(\hat{r} \frac{\partial \hat{u}_{z}}{\partial \hat{r}}\right)+\frac{\sigma}{\rho} \hat{B}^{2} U=0
\end{aligned}
$$

From which we observed that pressure does not vary in the radial $(\widehat{r})$, $\operatorname{circumferential}(\hat{\theta})$ and $\operatorname{axial}(\hat{z}) \mathrm{Z}$ direction and that pressure remain constant across any cross-section of the tube and $\hat{p}$ Is a function of the only $(\hat{z})$ that is $\hat{p}=p(\hat{z})$ and so pressure gradient term in the last equation above becomes $\frac{d \hat{p}}{d \hat{z}}$

Then (2)

$$
-\frac{1}{\rho} \frac{\partial \hat{p}}{\partial \hat{z}}+\frac{\mu}{\hat{r}} \frac{1}{\rho} \frac{\partial}{\partial \hat{r}}\left(\hat{r} \frac{\partial \hat{v}}{\partial \hat{r}}\right)+\frac{\sigma}{\rho} \hat{B}^{2} U=0
$$

Non-dimensional form

$$
\begin{aligned}
& r=\frac{\hat{r}}{R_{0}}, z=\frac{\hat{z}}{R_{0}}, R=\frac{\hat{R}}{R_{0}}, P=\frac{\hat{P}}{\rho U_{0}^{2}}, U=\frac{\hat{U}}{U_{0}}, B_{0}{ }^{2}=\frac{\hat{B}^{2}}{U_{0}}, \mu=\frac{\hat{\mu}}{\mu_{0}}, V=\frac{\mu \mu_{0}}{\rho} \\
& C=\frac{d \hat{p}}{d \hat{z}}=\frac{\rho U_{0}^{2}}{R_{0}} \frac{d p}{d z} \\
& C+\frac{1}{R_{e} r} \frac{d}{d r}\left(r \frac{d v}{d r}\right)+\frac{M^{2}}{R_{e}}=0
\end{aligned}
$$

SOLVING THE EQUATION (4):

$$
\begin{aligned}
& r^{2} \frac{d^{2} v}{d r^{2}}+r \frac{d v}{d r}=k r^{2} \\
& \text { Where } K=-\frac{C}{\mu} R_{e} r-\frac{M^{2} r}{\mu}
\end{aligned}
$$

Solution:

$$
\begin{gathered}
\text { Let } \quad r=e^{z}, D=\frac{d}{d z} \\
V=\frac{1}{4}\left[-\frac{C}{\mu} R_{e} r-\frac{M^{2} r}{\mu}\right] r^{2}
\end{gathered}
$$

Also, shear stress component at any distance $r$ from the tube axis is given by 


$$
\begin{gathered}
\tau_{r z}=\hat{\mu} \frac{d \hat{v}}{d \hat{r}}=\frac{\mu \mu_{0} U_{0}}{R_{0}} \frac{d v}{d r} \\
\tau_{r z}=\frac{\mu_{0} r U_{0}}{2 R_{0}}\left[-C R_{e}-M^{2}\right]
\end{gathered}
$$

Express for wall shear stress $\tau_{w}$ can be obtained from the formula

$$
\begin{aligned}
\tau_{w} & =\tau_{r z}(r=R) \\
\tau_{w} & =\frac{\mu_{0} R U_{0}}{2 R_{0}}\left[-C R_{e}-M^{2}\right]
\end{aligned}
$$

Using Equation (6) and $\quad \tau_{r z}\left(r_{c}\right)=\tau_{y}$ express for $\tau_{0}$ will lead to the form

$$
\tau_{y}=-\frac{r_{c} \mu_{0} U_{0}}{2}\left[-C R_{e}-M^{2}\right]
$$

In between $\tau_{Y}$ and $\tau_{w}$ there may arise two cases wall shear stress is greater and that yield stress. In case $\tau_{Y} \geq \tau_{w}$ that is if $r_{C} \geq R$ then there will occur no flow accordingly velocity function will become

$w_{Z}=0$

$w_{Z}=w_{Z}(r)$

Again, power fluid the constitutive equation may be reproduced in the following form

$\tau_{r z}=k \dot{v}^{n}$

$$
\tau_{r z} \geq \tau_{y}
$$

$\mathrm{K}$ is a constant and $\dot{v}$ is the scalar strain rate for the flow the strain rate can be expressed as $\dot{v}=-\frac{d v_{Z}}{d r}$ where $v_{i z}$ is the axial velocity

$$
k\left(\frac{d v_{z}}{d r}\right)^{n}=\frac{\mu_{0} r U_{0}}{2 R_{0}}\left[-C R_{e}-M^{2}\right]
$$

Integrate with respect to $\mathrm{r}$

$$
\begin{aligned}
& w=\frac{\mu_{0} r U_{0}}{2 R_{0}}\left[-C R_{e}-M^{2}\right]^{\frac{1}{n}}\left[\frac{R^{\frac{1+n}{n}}-r^{\frac{1+n}{n}}}{\frac{1+n}{n}}\right] \\
& w=\left\{\begin{array}{l}
w(r), r_{c} \leq r \leq R \\
w_{c}(r), 0 \leq r \leq r_{c} \\
\left.0, r_{c}\right\rangle R
\end{array}\right.
\end{aligned}
$$

The volume flux $\mathrm{Q}$ of the flow through the pipe is given by $Q=\int_{0}^{R} 2 \pi r w d r$

\section{Results And Discussion}

The objective of this analysis is to study the flow characteristics of a power fluid model for blood flow with velocity slip in presence of magnetic effect. The problem is solved numerically using Shooting method. Numerical calculations have been done for various considerations of parameters i.e., the Hartman Number M and the Reynolds number Re...Numerical results are shown graphically. It has been observed that the effect of the Hartmann number M and the Reynolds number Re on the velocity field as well as on the wall shear stress is very prominent. If shear stress $\tau_{r z}$ is greater than yield stress $\tau_{y}$ then a flow of blood is possible otherwise, there will be no blood flow.

Fig (1) shows that the nature of velocity profile is same if there is no slip.

Fig (2) Illustrate that the velocity profiles with an effect of a magnetic field for various values of Reynolds number. It is seen that the velocity profile decreases as the Reynolds number increases with slip.

Fig (3).Illustrate that the velocity profile $\mathrm{v}_{\mathrm{iz}}$ nature is not changed if no slip. The analysis developed here is based on certain assumptions which may lead to some physiological implications viz., (a) Flow is assumed steady which is indeed true for very thin arteries in CVS where the pulsatility effects are small. (b) The assumption that 
velocity variation in an axial direction is negligible as compared to its variation in radial direction, may lead to the implication that the length of the artery is too large as compared to the radius. Fig-4 Illustrate that the velocity profile $\mathrm{v}_{\mathrm{z}}$ decreases with increases of Hartmann number $\mathrm{M}$ in presence of slip. It is also observed that Hartmann Number increases the effect of magnetic field is decreases

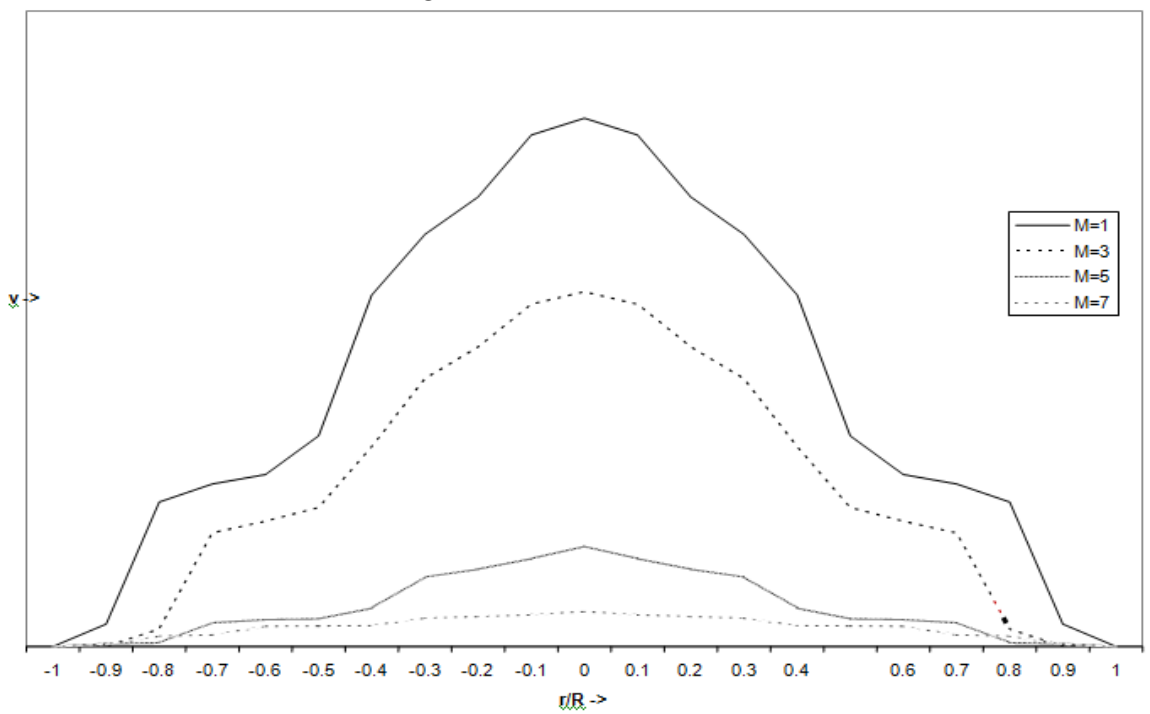

Fig. 1. Variation of Velocity profile for different Hartmann number at $\mathrm{Re}=\mathbf{2}$ with no slip

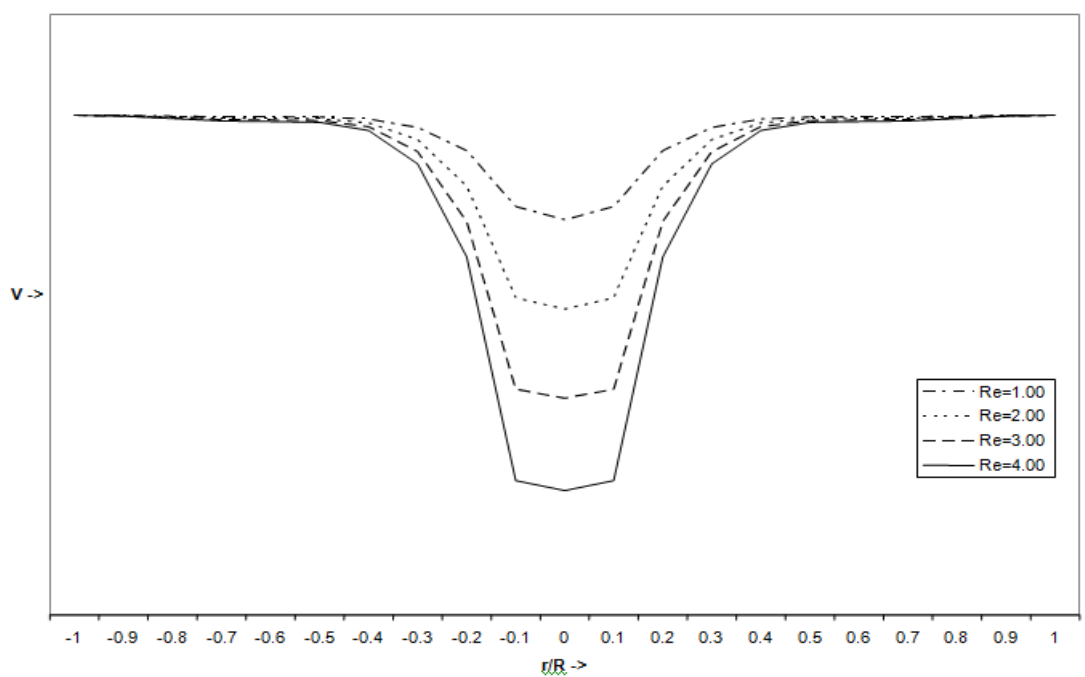

Fig. 2 Variation of velocity profile for different Reynolds number at $M=2$ with slip

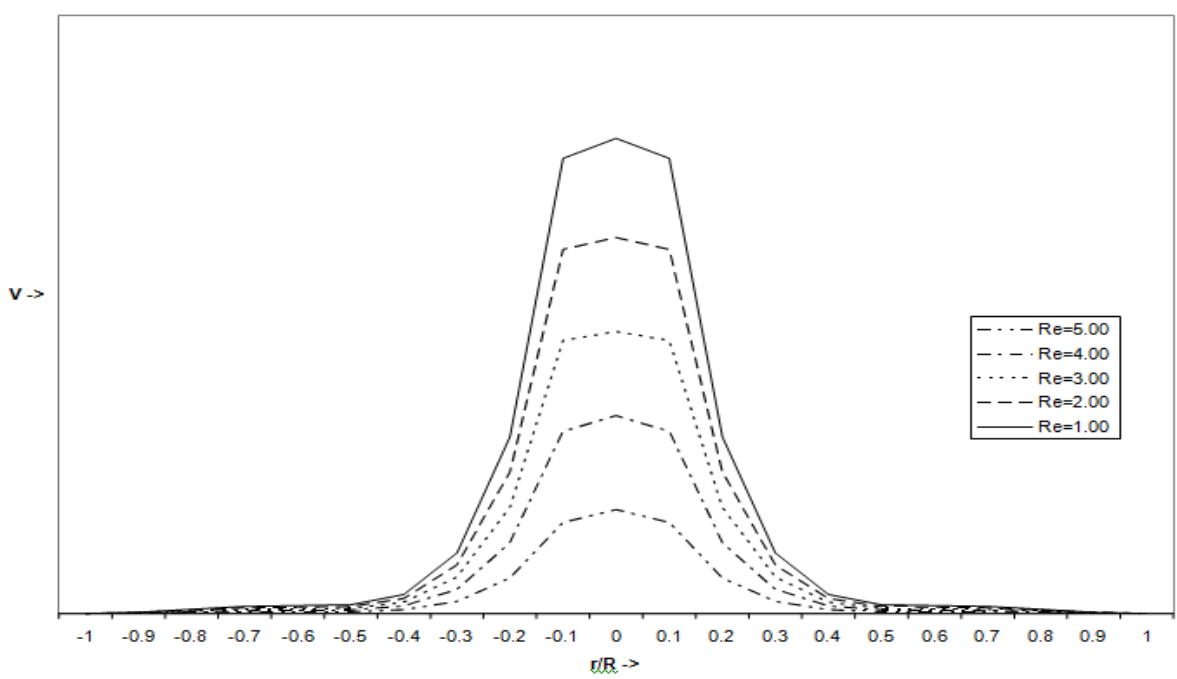

Fig 3. Variation of Velocity profiles for different Reynolds number at $M=2$ with no slip 


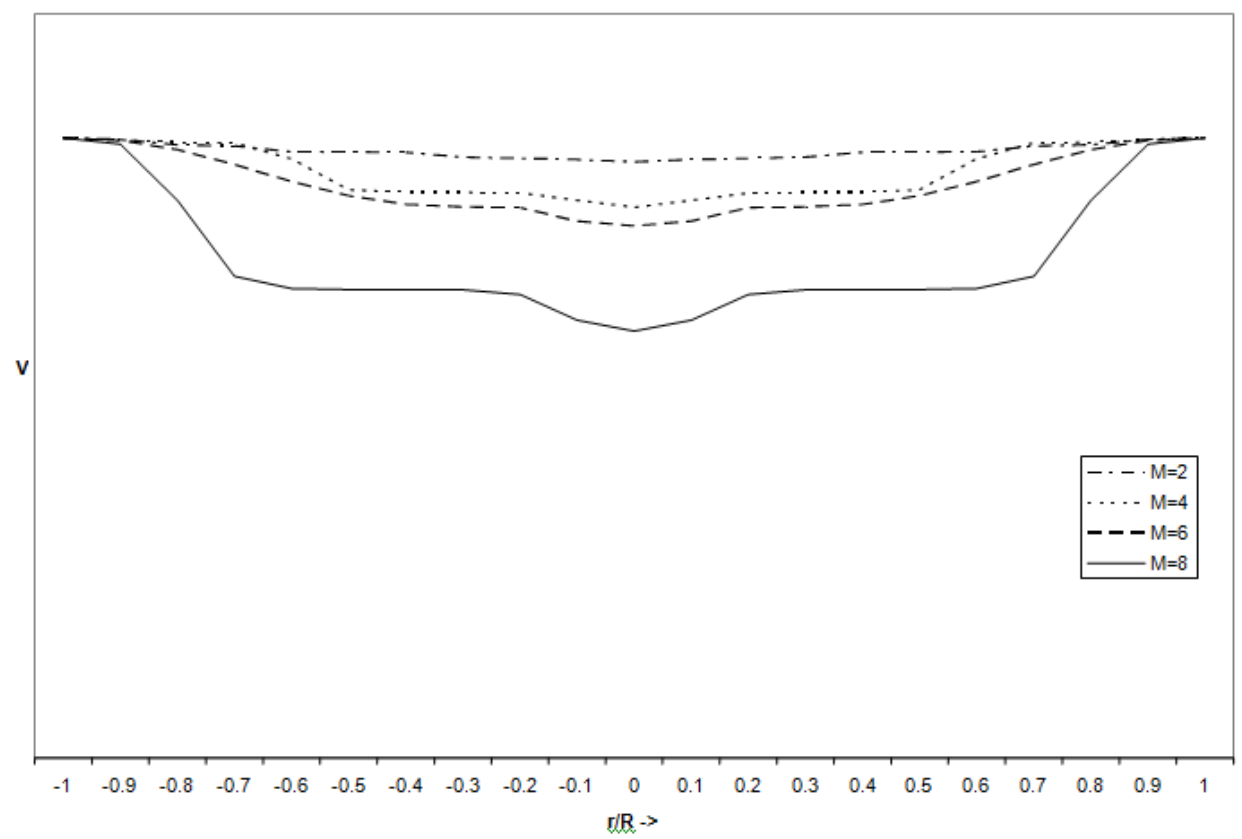

Fig. 4. Variation of Velocity profile for different Hartmann number at $\mathrm{Re}=\mathbf{2}$ with slip

\section{References}

[1]. Barnothy, M.F. (ed.), Biological effects of Magnetic field, Vol-I \& II, Plenum Press (1969).

[2]. Bhuyan, B.C and Hazarika, G.C, Bio-science Research Bulletin, Vol.17(2), PP.105-112(2001).

[3]. Bhuyan, B.C and Hazarika. G.C, proc. National conference on applied Mathematics, 212-217(2001).

[4]. Bhuyan B.C. and Hazarika G.C., Blood flow in channels of varying cross section with permeable boundaries in presence of transverse of magnetic field, Bio science Research Bulletin, Vol. 18 (No. 1), (2002)

[5]. Boyd, W., Text Book of Pathology: Structure and Functions in Diseases, Lea and Febigar, Philadelphia (1963).

[6]. Biswas, D and Nath , J., Oscillating Blood flow through a uniform Artery with wall slip, Journal of Assam University, 4(1), pp. 142-154(1999).

[7]. Brunn, P., The Velocity Slip of Polar Fluids, Rheol. Acta., 14, pp. 1039-1054 (1975).

[8]. Cocklet, G.R. The Rheology of humand blood, Biomechanics ed. Y.C. Fung, pp. 63. (1972)

[9]. Fung, Y. C. Biomechanics: Mechanical Properties of Living Tissues, Springer-Verlag, New York Inc. (1981).

[10]. M.E. Ali, N. Sandeep, Cattaneo-Christov model for radioactive heat transfer of magneto hydrodynamics Casson-Ferro fluid: A numerical study, Res.phy. 7(2017) 21-30.

[11]. M.Mustafa, T.Hayat, I.Pop, A.Aziz, Unsteady boundary layer flow of a Casson fluid due to an impulsively started moving flat plate, Heat Transf. Asian Res,40(2011) 563-576.

[12]. M.Y. Malik, M. Naseer, S. Nadeem, Abdul Rehman, The boundary layer flow of Cassonmonofluid over a vertical exponential stretching cylinder, Appl. Nanosci.4(2014).

[13]. MacDonald, D.A., On Steady Flow Through Modelled Vascular Stenoses, J. Biomechanics, 12, pp. 13-20 (1979)

[14]. M. Mustafa, J. Ahmad Khan, Model for flow of cassonnanofluid past a non-linearlystretching sheet considering magnetic field effects, AIP Adv. 5 (2015).

[15]. Merrill, F.W., Rheology of Human Blood and Some Speculations on its Role in Vascular Homeostatics Biomechanical Mechanisms in Vascular Homeostatics and Intravascular Thrombosis, ed. P.N. Sawyer, pp. 127-137, Appleton Century Crofts, New York (1965).

[16]. Nubar, Y., Blood Flow, Slip and Viscometry, Biophys., J., 11, pp. 252-264 (1971).

[17]. Sud, V.K. and Sekhon, G.S., Phy. Med. Biol., Vol. 34, pp. 795-805, (1989).

[18]. Rathod, V.P., Gayatri, Applied Science periodical, vol.2(1), PP, 52-58(2000).

[19]. Vardanyan, V.A., Biophysics, Vol. 18 (3), pp. 491-496 (1973)

[20]. Young,D.F ,J.Engng Ind.,Trans.Am.Soc.Mech.Engrs.Vol-90,p.248-254(1968) 